\title{
Prediction of unconventional protein secretion by exosomes
}

\author{
Alvaro Ras-Carmona, Marta Gomez-Perosanz and Pedro A. Reche
}

\section{${ }^{*}$ Correspondence:}

parecheg@med.ucm.es

Laboratory

of Immunomedicine

Department of Immunology

Faculty of Medicine,

Complutense University

of Madrid, Pza Ramón y Cajal,

$\mathrm{s} / \mathrm{n}, 28040$ Madrid, Spain

\begin{abstract}
Motivation: In eukaryotes, proteins targeted for secretion contain a signal peptide, which allows them to proceed through the conventional ER/Golgi-dependent pathway. However, an important number of proteins lacking a signal peptide can be secreted through unconventional routes, including that mediated by exosomes. Currently, no method is available to predict protein secretion via exosomes.
\end{abstract}

Results: Here, we first assembled a dataset including the sequences of 2992 proteins secreted by exosomes and 2961 proteins that are not secreted by exosomes. Subsequently, we trained different random forests models on feature vectors derived from the sequences in this dataset. In tenfold cross-validation, the best model was trained on dipeptide composition, reaching an accuracy of $69.88 \% \pm 2.08$ and an area under the curve (AUC) of $0.76 \pm 0.03$. In an independent dataset, this model reached an accuracy of $75.73 \%$ and an AUC of 0.840 . After these results, we developed ExoPred, a web-based tool that uses random forests to predict protein secretion by exosomes.

Conclusion: ExoPred is available for free public use at http://imath.med.ucm.es/exopr ed/. Datasets are available at http://imath.med.ucm.es/exopred/datasets/.

Keywords: Exosomes, Protein secretion, Random forests

\section{Background}

Protein secretion is of paramount relevance for cellular communication [1]. In eukaryotes, most secreted proteins follow the classical endoplasmic reticulum (ER)-Golgi pathway. This pathway requires the presence of a signal peptide in the $\mathrm{N}$-terminus of proteins (leader sequence), which promotes the delivery of nascent proteins into the lumen of the ER. Proteins are then transported to the Golgi apparatus and from there to the cellular surface via vesicular transport [2,3].

In addition, there are unconventional pathways of protein secretion, which actually enable the secretion of leaderless proteins. Unconventional secretion of proteins can occur through vesicular and non-vesicular pathways [3, 4]. In non-vesicular pathways, proteins are released directly to the extracellular space, while in vesicular pathways, proteins are released within vesicles. Cells can secrete to the extracellular environment a variety of vesicular structure, among which exosomes stand out [5].

(c) The Author(s), 2021. Open Access This article is licensed under a Creative Commons Attribution 4.0 International License, which permits use, sharing, adaptation, distribution and reproduction in any medium or format, as long as you give appropriate credit to the original author(s) and the source, provide a link to the Creative Commons licence, and indicate if changes were made. The images or other third party material in this article are included in the article's Creative Commons licence, unless indicated otherwise in a credit line to the material. If material is not included in the article's Creative Commons licence and your intended use is not permitted by statutory regulation or exceeds the permitted use, you will need to obtain permission directly from the copyright holder. To view a copy of this licence, visit http:// creativecommons.org/licenses/by/4.0/. The Creative Commons Public Domain Dedication waiver (http://creativecommons.org/publi cdomain/zero/1.0/) applies to the data made available in this article, unless otherwise stated in a credit line to the data. 
Exosomes are microvesicles ranging from 30 to $100 \mathrm{~nm}$ in size, playing an important role in intercellular communication thanks to their capacity to transport and transfer proteins, lipids and nucleic acids to other cells [6]. Exosome secretion has been involved in many biological processes, both in health and disease [7]. For example, exosomes are involved in the regulation of coagulation and inflammation $[6,8]$. Interestingly, the content of exosomes can change under pathological conditions such as cancer, neurodegenerative ailments and cardiovascular diseases $[7,9,10]$.

Exosomes are generated in the cytosol from inward budding invaginations of late endosomes, which results in intraluminal vesicles (ILV) within a large multivesicular body (MVB) [11]. When MVBs fuse with the plasma membrane, ILV are secreted into the extracellular space as exosomes $[2,12,13]$. Exosomes incorporate transmembrane proteins and a great variety of luminal cargo proteins, including cytosolic and nuclear proteins, lacking a signal peptide $[14,15]$.

Given the biological relevance of exosomes and their role in unconventional protein secretion, it is of great interest to identify and predict proteins secreted by these vesicles. Currently, there are several bioinformatics tools to predict proteins secreted through unconventional pathways [16], including SecretomeP [17], SPRED [18], SecretP $[19,20]$ and OutCyte [21]. All these tools are based on machine learning models that were not trained for the specific task of predicting protein secretion by exosomes. Here, we present ExoPred, a bioinformatic tool that is specific to predict proteins secreted by exosomes. ExoPred implements a Random Forests (RF) model that was trained on a sequence dataset including 2992 exosome luminal proteins assembled ex profeso. The dataset only included non-transmembrane and leaderless proteins from vertebrata. In tenfold cross-validation the model reached an accuracy of $69.88 \% \pm 2.08$ and an area under the curve (AUC) of $0.76 \pm 0.03$. Moreover, when tested in an independent dataset, this model reached an accuracy of $75.73 \%$.

\section{Results and discussion}

\section{Exosome training dataset}

Proteins secreted by exosomes are really diverse with regard to structure, function and sub-cellular location [22, 23]. Here, we aimed to predict proteins secreted within exosomes: luminal cargo proteins. To that end, we generated a non-redundant dataset containing 2992 proteins found within exosomes and 2961 that are not found in exosomes. In the dataset, we only considered highly curated proteins from vertebrates with less than $80 \%$ identity, discarding membrane bound proteins or carrying a leader sequence, as they could be secreted by other means (details in Methods). The average sequence identity between exosome proteins included in the training dataset is $12.22 \pm 2.02(\%)$, while the sequence identity between non-exosome proteins is $12.23 \pm 2.38$ (\%). Overall, the average sequence identity in the training dataset is $12.19 \pm 2.08$ (\%). In Additional file 1: Figure S1, we show additional measures of identity in the training dataset.

We investigated the sub-cellular location of exosome proteins upon UNIPROT annotations as a mean to select appropriated non-exosome proteins for inclusion in the training dataset (details in Methods). As shown in Fig. 1a, some exosome proteins have no sub-cellular location annotations (456) while many others (1225) have more than one 


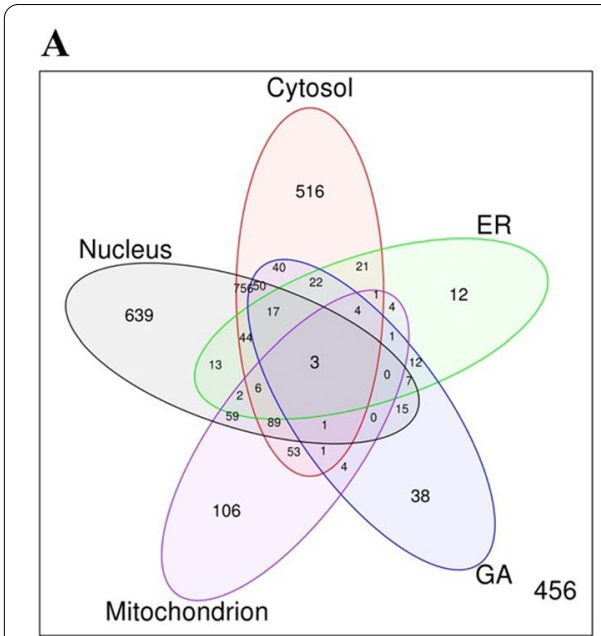

B

Fig. 1 Sub-cellular location of proteins included in the training dataset. Venn diagrams indicating the sub-cellular location of exosome (a) and non-exosome proteins (b) as annotated in UNIPROT. Note that the preferential sub-cellular location for exosome and non-exosome proteins was cytosolic and nuclear

sub-cellular location. The dataset also includes 639, 516, 12, 38 and 106 exosome proteins that are annotated with exclusive sub-cellular locations in the nucleus, cytosol, ER, GA and mitochondrion, respectively. Likewise, non-exosome proteins included in the training dataset exhibit sub-cellular locations that mirror those of exosome proteins (Fig. 1b). By including in the training dataset exosome and non-exosome proteins with balanced sub-cellular locations, we aimed to obtain prediction models that were robust and unbiased.

\section{Generation and evaluation of RF models predicting proteins secreted by exosomes}

Luminal exosome proteins are very heterogeneous and, unlike proteins secreted by the classical secretion pathway, do not have any recognizable pattern determining their secretion by exosomes. Under this scenario, machine learning techniques provide a suitable approach to predict proteins secreted by exosomes. In this work, we specifically used RF, as they have been shown to exhibit high prediction accuracy in many biological problems and with different types of data [24]. Moreover, RF are more intuitive and have less parameters that need optimization than other machine learning algorithms such as support vector machines, which are often applied in classification [25].

To generate prediction models, we trained and evaluated various RF under tenfold cross-validation classification experiments (details in Methods) in the described training dataset translated into feature vectors consisting of amino acid composition, physico-chemical properties, dipeptide composition, the combination of amino acid composition plus physico-chemical properties and the combination of all of them (details in Methods). We chose these features since they can be obtained from the sequences alone. Hereinafter, we will refer to the RF models generated to predict protein secretion by exosomes as esRF. As shown in Fig. 2, in all sequence feature vectors the accuracy of esRF improved as the interaction value increased, stabilizing approximately at a value of 1500 . An esRF model trained on dipeptide composition with an 


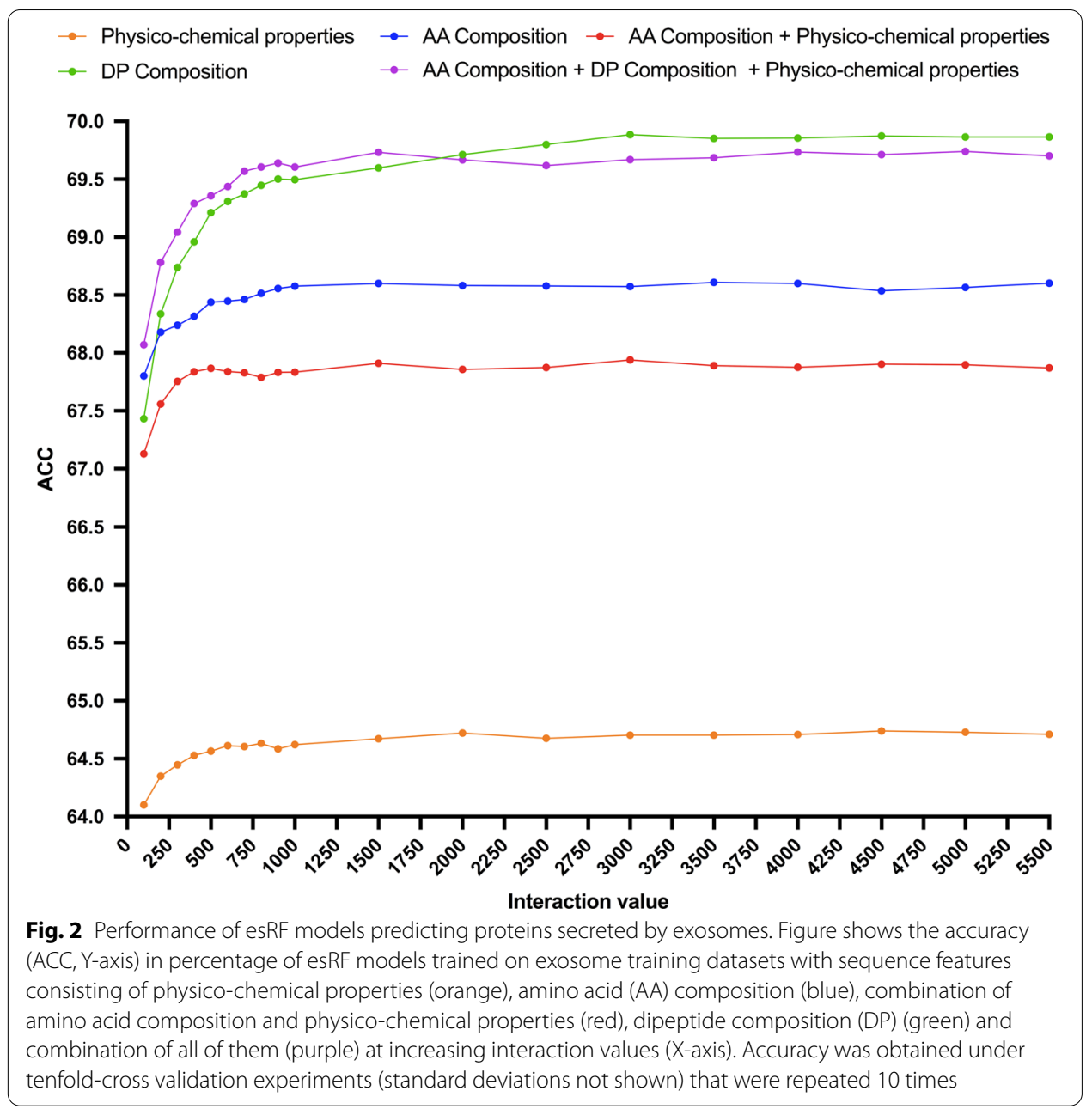

interaction value of 3000 reached the top classification accuracy, $69.88 \% \pm 2.08$. This esRF model produced classifications with AUC of $0.76 \pm 0.03$ and MCC of $0.40 \pm 0.05$ (Table 1). Note that training esRF models on additional features to dipeptide composition, such as physico-chemical properties and amino acid composition, did not improve the accuracy of the predictions.

Table 1 Performance of top esRF models generated in this study

\begin{tabular}{llllll}
\hline \multicolumn{5}{l}{ Dataset translation } \\
\cline { 2 - 6 } & PCP & AA & PCP + AA & DP & PCP + AA + DP \\
\hline I & 4500 & 3500 & 3000 & 3000 & 5000 \\
ACC (\%) & $64.74 \pm 1.94$ & $68.61 \pm 2.05$ & $67.94 \pm 2.02$ & $69.88 \pm 2.08$ & $69.74 \pm 1.93$ \\
AUC & $0.69 \pm 0.04$ & $0.75 \pm 0.03$ & $0.72 \pm 0.06$ & $0.76 \pm 0.03$ & $0.76 \pm 0.04$ \\
MCC & $0.29 \pm 0.06$ & $0.37 \pm 0.04$ & $0.36 \pm 0.05$ & $0.40 \pm 0.05$ & $0.39 \pm 0.05$ \\
\hline
\end{tabular}

Table reports the accuracy (ACC), area under the curve (AUC) and Matthews correlation coefficient (MCC) of the top performing esRF models obtained with the indicated interaction value (I) by training in physico-chemical properties $(P C P)$, amino acid composition (AA), combination of physico-chemical properties and amino acid composition (PCP $+A A)$, dipeptide composition (DP) and combination of physico-chemical properties, amino acid composition and dipeptide composition (PCP + AA + DP). Performance values were obtained under 10-cross validation experiments that were repeated 10 times 
The features used for training have a major impact in the performance of the models and we cannot discard the potential benefit of having considered more complex and/or additional features like gene ontology (GO) [26], evolutionary information [27, 28] and/or protein profile-alignments [29]. The inclusion of evolutionary and profile-alignment features is an ingenious manner to enhance datasets and it is of particularly interest when instances for training are limited. However, the use of these types of features, in particular those based on profile-alignments, can cloud former efforts to decrease sequence similarity in the training datasets and demands much computational power. The use of knowledge-based annotations such as GO terms has shown promising results in protein classification problems $[26,30]$. However, these annotations are not readily available for all the proteins and will need to be predicted or skipped, thus hampering the utility of the resulting models. Combining different features like those mentioned above could also lead to gains in performance but model overfitting becomes a concern when training in numerous features [31]. Moreover, combining complex features is not trivial and can impact the performance of the models $[32,33]$. Therefore, in this work we trained and evaluated models on simple and few sequence-features that could be easily extracted and combined.

To further assess the predictive power of top esRF models selected in cross-validation, we tested them in an independent test dataset consisting of 2346 exosome proteins and 3443 non-exosome proteins generated as indicated in Methods. The similarity between the training and the independent test dataset was very low; overall the average sequence identity between the two datasets was of $12.01 \pm 3.30$ (\%). More measures of identity between these two datasets are provided in Additional file 1: Figure S1. For testing, the independent dataset was translated into feature vectors matching the relevant esRF and their performance is shown in Table 2. All esRF models were able to discriminate proteins secreted by exosomes better than in crossvalidation exhibiting an ACC over $68 \%$. The largest ACC was reached again by the esRF model trained on dipeptide composition ( $\mathrm{ACC}=75.73 \%)$. The fact that the performance of esRF models in the independent test dataset was better than in crossvalidation along with the larger number of sequences used for model building sharing little sequence similarity supports the robustness of our esRF models to predict proteins secreted by exosomes.

Table 2 Performance of esRF models in an independent testing dataset

\begin{tabular}{llllll}
\hline \multirow{5}{*}{} & \multicolumn{4}{l}{ Dataset translation } \\
\cline { 2 - 6 } & PCP & AA & PCP + AA & DP & PCP + AA + DP \\
\hline ACC (\%) & 68.99 & 71.49 & 71.41 & 75.73 & 75.54 \\
AUC & 0.765 & 0.793 & 0.795 & 0.840 & 0.839 \\
MCC & 0.378 & 0.424 & 0.422 & 0.505 & 0.503 \\
\hline
\end{tabular}

Table reports the accuracy (ACC \%), area under the curve (AUC) and Matthews correlation coefficient (MCC) reached in the independent dataset by the top performing esRF model obtained by training in sequence features consisting of physicochemical properties (PCP), amino acid composition (AA), combination of global properties and amino acid composition $(\mathrm{PCP}+\mathrm{AA})$, dipeptide composition (DP) and all of them (PCP + AA + DP) 


\section{Comparison of esRF with related methods}

Currently, there is not any specific tool to predict protein secretion by exosomes. However, there are a few bioinformatics tools aimed to predict proteins secreted by unconventional pathways [16], and among them, we selected SecretomeP [17] and OutCyte [21], which are both available for free public use over the internet, for comparison with our method. SecretomeP is based on neural networks trained on a dataset of 3654 mammalian proteins that are secreted by unconventional pathways, including exosome cargo proteins [17], and it is often considered as a reference tool. OutCyte is a novel tool related with SecretomeP, also based on neural networks, but yielding contrasting predictions when tested in the human proteome [21].

We evaluated the SE, SP and ACC of SecretomeP and OutCyte in our independent dataset and compared the results with those obtained by our top esRF model trained on dipeptide composition. As shown in Table 3, our esRF model produced values of SE and SP of 0.73 and 0.78 respectively, both clearly superior to those obtained with SecretomeP and OutCyte. The ACC of our esRF model in the test dataset was also clearly superior to that of SecretomeP and OutCyte (75.73\% vs $45.14 \%$ and $54.39 \%$, respectively). Overall, these results indicate that SecretomeP and OutCyte are surprisingly unable to predict proteins secreted by exosomes, which highlights the utility of the esRF models developed here.

It is worth nothing that exosome secretion of proteins could have been approached as a sub-cellular location problem. However, the fact that proteins in exosomes can also have different locations complicates this approach. A suitable solution, already applied to predict sub-cellular location of proteins, would be to train classification models considering multi-labels $[26,29,30]$. However, to our knowledge, not even the most recent methods of sub-cellular location prediction consider exosomes within their predicted locations [26-30, 32-34]. Therefore, we suggest combining our models of exosome secretion with those that can predict sub-cellular location.

Table 3 Comparative performance of Outcyte, SecretomeP and esRF

\begin{tabular}{lccr}
\hline & \multicolumn{2}{c}{ Prediction method } & \\
\cline { 2 - 4 } & OutCyte & SecretomeP & esRF \\
\hline SE & 0.32 & 0.25 & 0.73 \\
SP & 0.72 & 0.65 & 0.78 \\
ACC (\%) & 54.39 & 45.14 & 75.73 \\
\hline
\end{tabular}

SecretomeP, OutCyte and esRF models were evaluated in our independent dataset and compared with regard to sensitivity (SE), specificity (SP) and accuracy (ACC). SecretomeP predictions were obtained at http://www.cbs.dtu.dk/services/Secre tomeP/ selecting the mammalian option and the default prediction threshold of 0.6 . OutCyte predictions were obtained at http://www.outcyte.com selecting the "OutCyte-UPS" model and default settings

\section{ExoPred web server}

Given the results described above and the relevance of predicting proteins secreted by exosomes, we developed a web-based tool, ExoPred, which implements our top esRF for free public use (http://imath.med.ucm.es/exopred/). The ExoPred interface, shown in Fig. 3a, has been designed for simple and intuitive use. The input data for ExoPred can be one or several protein sequences in FASTA format, which can be pasted or uploaded 
A

Immunomedicine Group

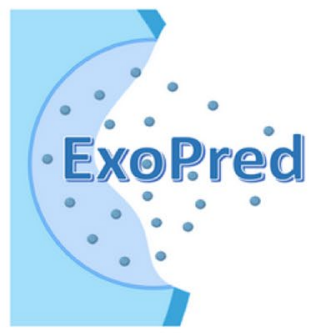

Prediction of Exosome Protein Secretion

\begin{tabular}{l}
\hline User Guide \\
Example Output \\
vehicles for the secretion of proteins without a signal peptide. Proteins secreted by exosomes are \\
very heterogeneous and lack any sequence pattern facilitating their recognition. ExoPred has been \\
verecifically developed to identify proteins secreted by exosomes in vertebrates. ExoPred \\
predictions are based on a random forest model trained on dipeptide composition of exosome
\end{tabular}
proteins that achieved $75.73 \%$ accuracy in an independent dataset

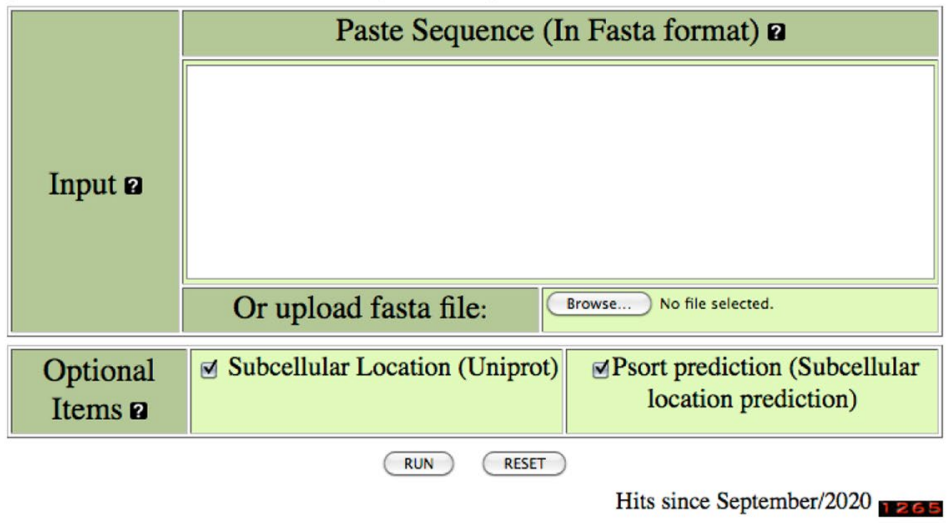

B

\section{RESULTS}

\begin{tabular}{|c|c|c|c|c|c|c|c|c|}
\hline Seq \# & Protein Name & Vertebrata & Protein ID I & Leader Seq & Transmembrane & Uniprot Subcellular location & Psort Prediction & ExoPred \\
\hline 1 & 7B2_PIG & $\mathrm{Y}$ & P01165.2 & $\mathrm{Y}$ & $\mathrm{N}$ & Extracellular & Extracellular, including cell wall & $1 \mathrm{NA}$ \\
\hline 2 & CD81_CHLAE & $\mathrm{Y}$ & 097703.1 & $\mathrm{N}$ & $\mathrm{Y}$ & Plasma Membrane & Endoplasmic reticulum & NA \\
\hline 3 & TKFC_HUMAN & $\mathrm{Y}$ & Q3LXA3.2 & $\mathrm{N}$ & $\mathbf{N}$ & Nucleus; Cytosol; Extracellular; EXOSOME & Cytoplasmic & $\mathrm{Y}$ \\
\hline 4 & RN5A_HUMAN & $\mathrm{Y}$ & Q05823.2 & $\mathrm{N}$ & $\mathrm{N}$ & Nucleus; Cytosol; Mitochondrion & Mitochondrial & $\mathrm{N}$ \\
\hline
\end{tabular}

Fig. 3 ExoPred web server. a ExoPred interface. b ExoPred result page with sub-cellular and PSORT predictions selected. The information shown is the following (from left to right): Number of the sequence; name of the sequence in the FASTA file; belonging to vertebrates (Y/N); UNIPROT ID; presence of predicted signal peptides (Y/N) or transmembrane regions (Y/N); sub-cellular location annotated in UNIPROT; sub-cellular location predicted by PSORT [35]; and, finally, exosome secretion prediction (Y/N)

to the server. In ExoPred, users can also select to retrieve the sub-cellular location of input proteins as annotated in UNIPROT and/or predict such sub-cellular location using PSORT (version II) [35]. After submission, ExoPred first runs a BLASTP [36] against the UNIPROT database and processes the BLAST output to identify the UNIPROT identifier (ID) of protein hits with identity higher than $90 \%$ and over $90 \%$ of their entire length. After these identifiers, ExoPred will then retrieve taxa and sub-cellular location information from UNIPROT annotations and transfer it to the relevant input query proteins. 
ExoPred will also detect those proteins with leader sequences and transmembrane regions using SignalP [37] and TMHMM [38] and predict sub-cellular locations using PSORT [35].

The model for predicting exosome secretion is only executed in proteins from vertebrate and without a signal peptide or transmembrane regions. ExoPred output consists of a table reporting by default whether input proteins are from vertebrate $(\mathrm{Y} / \mathrm{N})$, contain a signal peptide $(\mathrm{Y} / \mathrm{N})$ or transmembrane regions $(\mathrm{Y} / \mathrm{N})$ and can be secreted via exosomes $(\mathrm{Y} / \mathrm{N})$. As show in Fig. 3b, ExoPred will also show the sub-cellular location of input proteins annotated in UNIPROT and predicted by PSORT if the relevant options were checked at submission. Exosome secretion predictions will show as NA (not available) for input proteins that do not meet the criteria mentioned above. For proteins without UNIPROT equivalents, ExoPred will still determine whether they can be secreted by exosomes as long as they have no predicted signal peptide or transmembrane regions. In these cases, the field taxa, and UNIPROT sub-cellular-location, when selected, will show as not found.

\section{Conclusions}

Exosomes have a relevant role in intercellular communication in eukaryotes, representing a major vehicle to secret leaderless proteins. Currently, no method is available to specifically predict protein secretion by exosomes. Here, we developed ExoPred, a webbased tool to predict proteins secreted by exosomes. ExoPred predictions are based on random forests models that achieved $75.73 \%$ accuracy in an independent dataset. Predicting and annotating that a particular protein can be secreted by exosomes is clearly relevant, as it is indicative of a potential role in cell communication and suggests new untapped functions. Therefore, we plan to release a standalone version for resources and bioinformaticians providing protein sequence annotations.

\section{Methods}

\section{Generation of exosome protein datasets}

In this study, we assembled two non-overlapping protein datasets, a training dataset and an independent test dataset, both including exosome and non-exosome proteins. Exosome proteins in the training dataset where obtained from QuickGo [39] after the GO term "extracellular exosome" ([GO: 0070062]) and from ExoCarta database [40]. Nonvertebrate proteins and ExoCarta proteins without UNIPROT [41] representation were not considered as well as unreviewed proteins and those with a global annotation score lower than 3 out of 5 as annotated by UNIPROT. Likewise, exosome proteins including signal peptides and/or transmembrane regions were discarded. CD-HIT software [42] was applied to reduce sequence similarity so that exosome proteins in the training dataset do not share more than $80 \%$ identity. Non-exosome proteins in the training dataset were randomly collected from UNIPROT and obeyed to the same criteria than exosome proteins (reviewed, from vertebrate, global annotation $\geq 3$, sequence similarity under $80 \%$ and exclusion of proteins with a leader sequence and/or transmembrane regions). Exosome proteins in the test dataset met the same criteria than those in the training dataset but were obtained from ExoCarta without considering UNIPROT quality 
annotations. Non-exosome proteins in the test dataset were also obtained as described earlier. Datasets are available at http://imath.med.ucm.es/exopred/datasets/.

\section{Sequence similarity analyses}

Sequence similarity in training and independent datasets was analyzed after pairwise sequence alignments generated using the Needleman-Wunsch global alignment algorithm implemented by the needle application of the EMBOSS package [43]. To obtain a measure of sequence similarity in a dataset, all sequences were aligned pairwise but with themselves (for a dataset with $N$ sequences there will be $N \times N-1$ alignments), identities were obtained for each alignment and the average identity was computed.

\section{Model building and evaluation}

Models to predict proteins secreted by exosomes were built using the Waikato Environment for Knowledge Analysis (WEKA) package [44]. WEKA provides a framework for data classification, clustering and feature selection using a large collection of machine learning algorithms. In WEKA, exosome protein secretion models were trained and evaluated under the application EXPLORER, using RF as classification algorithms. Classification with RF operate by applying multiple decision trees generated during training and outputting a modal decision $[45,46]$. Different RF models were obtained by varying the interaction value of the algorithm $(100,150,200,250,300,350,400,450,500$, $550,600,650,700,750,800,850,900,950,1000,1500,2000,2500,3000,3500,4000$, $4500,5000,5500,6000,8000,10,000)$. The interaction value indicates the number of trees in RF. As input for WEKA, we used five distinct training datasets in ARFF format resulting of translating the amino acid sequences in the exosome training dataset into feature vectors of equal length. Sequence feature vectors consisted of amino acid composition, physico-chemical properties, dipeptide composition, the combination of amino acid composition and physico-chemical properties, and the combination of all of them. Amino acid and dipeptide compositions of protein sequences were computed as described elsewhere [47]. The amino acid composition feature vector contains 20 values indicating the proportion of each of the 20 natural amino acids in the sequence. The dipeptide composition feature vector contains 400 values depicting the proportion of all possible pair of amino acids $(20 \times 20)$ in the sequences. The physico-chemical property feature vector contains 11 values, $P_{\dot{b}}$ computed for each sequence after 11 distinct amino acid properties. For each physico-chemical property, $i, P_{i}$, was computed using Eq. 1 where $p_{i} a_{\mathrm{n}}$ is the relevant normalized physico-chemical property of amino acid, $a$, at the $n$ position of a given protein sequence, and $N$ is the total number of amino acid residues in the sequence.

$$
P_{i}=\frac{\sum_{n=1}^{N} p_{i} a_{n}}{N}
$$

The 11 amino acid physico-chemical properties used in this study included average flexibility indices [48], residue volume [49], relative mutability [50], net charge [51], optimized side chain interaction parameter [52], polarity [53], alpha-helix propensity derived from designed sequences [54], beta-sheet propensity derived from designed sequences [54], amphiphilicity index [55], modified Kyte-Doolittle hydrophobicity scale 
[56] and aromaticity. Combination feature vectors were obtained by merging the relevant vectors. As a result, the amino acid composition and physico-chemical property feature vector contains 33 values resulting of merging 20 amino acid composition values and 11 physico-chemical property values per sequence into a single vector. Likewise, the combined feature vector containing amino acid and dipeptide compositions, and physico-chemical properties contains 433 values.

RF models were trained and evaluated in tenfold cross-validation classification experiments that were repeated 10 times. Best performing models that were obtained by training in the noted sequence features were also evaluated in the test dataset.

\section{Measures of performance}

The performance of RF models was obtained by computing threshold-dependent measures such as sensitivity (SE), specificity (SP), Matthews correlation coefficient (MCC) and accuracy (ACC) using Eqs. 2, 3, 4 and 5, respectively. These measurements are expressed in terms of true positive (TP), false negative (FN), true negative (TN) and false positive (FP) predictions.

$$
\begin{aligned}
& S E=\frac{T P}{T P+F N} \\
& S P=\frac{T N}{T N+F P} \\
& M C C=\frac{(T P \times T N)-(F N \times F N)}{\sqrt{(T N+F N)(T P+F N)(T N+F P)(T P+F P)}} \\
& A C C=\frac{(T P+T N)}{(T P+F P+T N+F N)} \times 100
\end{aligned}
$$

The performance of RF models was also evaluated by computing the area under the curve (AUC) resulting from plotting SE vs 1-SP at different thresholds. An AUC value of 0.5 corresponds to a random prediction, while a value of 1 reflects a perfect prediction.

\section{Prediction of unconventional protein secretion using SecretomeP and OutCyte}

SecretomeP [17] is a web-based tool for predicting unconventional protein secretion available at http://www.cbs.dtu.dk/services/SecretomeP/. For comparative analysis, SecretomeP was used to predict exosome and non-exosome proteins in the test dataset selecting the "Mammalian" option. Proteins with "NN-scores" higher than 0.6 were considered as secreted by an unconventional pathway, as indicated in the web page. OutCyte [21] is another web-tool, available at http://www.outcyte.com, for the prediction of unconventional protein secretion based on convolutional neural networks. For comparative analysis, proteins included in the test dataset were subjected to OutCyte predictions, using the model "OutCyte-UPS" with the default settings. 


\section{Web implementation}

Exosome prediction models were implemented for free public use on the Web using a Python CGI (Common Gateway Interface) script that executes the predictions on userprovided input data and returns the results to the browser. The front-end web interface was developed using Hyper Text Markup Language (HTML) in combination with Cascading Style Sheets (CSS) and JavaScript. Web page administration is done using Apache HTTP Server (https://httpd.apache.org).

\section{Abbreviations}

AA: Amino acid composition; ACC: Accuracy; AUC: Area under the curve; DP: Dipeptide composition; ER: Endoplasmic reticulum; GA: Golgi aparatus; MCC: Mathew's correlation coefficient; PCP: Physico-chemical properties; RF: Random forests; SE: Sensitivity; SP: Specificity.

\section{Supplementary Information}

The online version contains supplementary material available at https://doi.org/10.1186/s12859-021-04219-z.

Additional file 1: Figure S1. Sequence similarity in training and testing datasets. The figure shows the percentage of identity between exosome proteins, non-exosome proteins, exosome vs non-exosome proteins and all sequences found in the training datasets and independent test datasets. It also shows the percentage of identity between proteins of the training dataset with those of the independent test dataset considering exosome proteins, nonexosome proteins, exosome proteins vs non-exosome proteins and all proteins. Sequence identity was computed as indicated in Methods and reported as an average identity with their standard deviation.

\section{Acknowledgements}

We wish to acknowledge Dr. Lafuente for critical reading and helpful comments.

\section{Authors' contributions}

Conceptualization: P.A.R; Methodology: M.G.P, A.R.C \& P.A.R; Writing-Original Draft: A.R.C \& P.A.R; Final Writing \& Editing: A.R.C, P.A.R \& M.G.P. All authors have read and approved the final manuscript.

Funding

Not applicable.

Availability of data and materials

Authors confirm that all relevant data are included in the article.

\section{Declarations}

Ethics approval and consent to participate

Not applicable.

Consent for publication

Not applicable.

\section{Competing interests}

All the authors declare that they have no competing interests.

Received: 15 March 2021 Accepted: 21 May 2021

Published online: 16 June 2021

\section{References}

1. Chen Z, Chen JJ, Fan R. Single-cell protein secretion detection and profiling. Annu Rev Anal Chem. 2019;12:431-49. https://doi.org/10.1146/annurev-anchem-061318-115055.

2. Lopez-Verrilli MA, Court FA. Exosomes: mediators of communication in eukaryotes. Biol Res. 2013;46:5-11.

3. Rabouille C, Malhotra V, Nickel W. Diversity in unconventional protein secretion. J Cell Sci. 2012;125:5251 LP - 5255. doi:https://doi.org/10.1242/jcs.103630.

4. Nickel W. Pathways of unconventional protein secretion. Current Opinion in Biotechnology. 2010.

5. Ng F, Tang BL. Unconventional protein secretion in animal cells BT - unconventional protein secretion: methods and protocols. In: Pompa A, De Marchis F, editors. New York, NY: Springer New York; 2016. p. 31-46. doi:https://doi.org/ 10.1007/978-1-4939-3804-9_2.

6. Yáñez-Mó M, Siljander PR-M, Andreu Z, Zavec AB, Borràs FE, Buzas El, et al. Biological properties of extracellular vesicles and their physiological functions. J Extracell vesicles. 2015;4:27066. doi:https://doi.org/10.3402/jev.v4.27066. 
7. Bang C, Thum T. Exosomes: new players in cell-cell communication. Int J Biochem Cell Biol. 2012;44:2060-4. https:// doi.org/10.1016/j.biocel.2012.08.007.

8. Di Pietro C. Exosome-mediated communication in the ovarian follicle. J Assist Reprod Genet. 2016;33:303-11. https://doi.org/10.1007/s10815-016-0657-9.

9. Milane L, Singh A, Mattheolabakis G, Suresh M, Amiji MM. Exosome mediated communication within the tumor microenvironment. J Controlled Release. 2015.

10. Li A, Zhang T, Zheng M, Liu Y, Chen Z. Exosomal proteins as potential markers of tumor diagnosis. J Hematol Oncol. 2017;10:175. https://doi.org/10.1186/s13045-017-0542-8.

11. Zhang Y, Liu Y, Liu H, Tang WH. Exosomes: biogenesis, biologic function and clinical potential. Cell Biosci. 2019;9:19. https://doi.org/10.1186/s13578-019-0282-2.

12. Kowal J, Tkach M, Théry C. Biogenesis and secretion of exosomes. Curr Opin Cell Biol. 2014;29:116-25. https://doi. org/10.1016/j.ceb.2014.05.004.

13. Schorey JS, Cheng Y, Singh PP, Smith VL. Exosomes and other extracellular vesicles in host-pathogen interactions. EMBO Rep. 2015;16:24-43. https://doi.org/10.15252/embr.201439363.

14. Rashed MH, Bayraktar E, Helal GK, Abd-Ellah MF, Amero P, Chavez-Reyes A, et al. Exosomes: from garbage bins to promising therapeutic targets. Int J Mol Sci. 2017;18:538. doi:https://doi.org/10.3390/ijms18030538.

15. Kalra H, Drummen GPC, Mathivanan S. Focus on extracellular vesicles: introducing the next small big thing. Int J Mol Sci. 2016;17:170. https://doi.org/10.3390/ijms17020170

16. Nielsen H, Petsalaki El, Zhao L, Stühler K. Predicting eukaryotic protein secretion without signals. Biochim Biophys Acta Proteins Proteomics. 2019:1867:140174.

17. Bendtsen JD, Jensen $L$, Blom N, von Heijne G, Brunak S. Feature-based prediction of non-classical and leaderless protein secretion. Protein Eng Des Sel. 2004;17:349-56. https://doi.org/10.1093/protein/gzh037.

18. Kandaswamy KK, Pugalenthi G, Hartmann E, Kalies K-U, Möller S, Suganthan PN, et al. SPRED: a machine learning approach for the identification of classical and non-classical secretory proteins in mammalian genomes. Biochem Biophys Res Commun. 2010;391:1306-11. https://doi.org/10.1016/J.BBRC.2009.12.019.

19. Yu L, Guo Y, Zhang Z, Li Y, Li M, Li G, et al. SecretP: A new method for predicting mammalian secreted proteins. Peptides. 2010;31:574-8. https://doi.org/10.1016/J.PEPTIDES.2009.12.026.

20. Yu L, Guo Y, Li Y, Li G, Li M, Luo J, et al. SecretP: Identifying bacterial secreted proteins by fusing new features into Chou's pseudo-amino acid composition. J Theor Biol. 2010;267:1-6. https://doi.org/10.1016/J.JTBI.2010.08.001.

21. Zhao L, Poschmann G, Waldera-Lupa D, Rafiee N, Kollmann M, Stühler K. OutCyte: a novel tool for predicting unconventional protein secretion. Sci Rep. 2019;9:19448. https://doi.org/10.1038/s41598-019-55351-z.

22. Pegtel DM, Gould SJ. Exosomes. Annu Rev Biochem. 2019;88:487-514. https://doi.org/10.1146/annurev-bioch em-013118-111902.

23. Mathieu M, Martin-Jaular L, Lavieu G, Théry C. Specificities of secretion and uptake of exosomes and other extracelIular vesicles for cell-to-cell communication. Nat Cell Biol. 2019;21:9-17. https://doi.org/10.1038/s41556-018-0250-9.

24. Qi Y. Random Forest for Bioinformatics BT - Ensemble Machine Learning: Methods and Applications. In: Zhang C, Ma Y, editors. Boston, MA: Springer US; 2012. p. 307-23. doi:https://doi.org/10.1007/978-1-4419-9326-7_11.

25. Martínez-Naves E, Lafuente EM, Reche PA. Recognition of the ligand-type specificity of classical and non-classical MHC I proteins. FEBS Lett. 2011;585:3478-84.

26. Wan S, Mak M-W, Kung S-Y. HybridGO-Loc: mining hybrid features on gene ontology for predicting subcellular localization of multi-location proteins. PLoS ONE. 2014;9:e89545. https://doi.org/10.1371/journal.pone.0089545.

27. Uddin MR, Sharma A, Farid DM, Rahman MM, Dehzangi A, Shatabda S. EvoStruct-sub: an accurate gram-positive protein subcellular localization predictor using evolutionary and structural features. J Theor Biol. 2018;443:138-46.

28. Xiang $\mathrm{Q}$, Liao $\mathrm{B}, \mathrm{Li}$ X, Xu H, Chen J, Shi Z, et al. Subcellular localization prediction of apoptosis proteins based on evolutionary information and support vector machine. Artif Intell Med. 2017;78:41-6.

29. Wan S, Mak M-W, Kung S-Y. Transductive learning for multi-label protein subchloroplast localization prediction. IEEE/ ACM Trans Comput Biol Bioinforma. 2017;14:212-24.

30. Lin W-Z, Fang J-A, Xiao X, Chou K-C. iLoc-Animal: a multi-label learning classifier for predicting subcellular localization of animal proteins. Mol Biosyst. 2013;9:634-44.

31. Tang J, Alelyani S, Liu H. Feature selection for classification: a review. Data Classification: Algorithms and Applications. 2014;:37-64. doi:https://doi.org/10.1201/b17320.

32. Wan S, Mak M-W, Kung S-Y. Ensemble linear neighborhood propagation for predicting subchloroplast localization of multi-location proteins. J Proteome Res. 2016;15:4755-62.

33. Wei L, Liao M, Gao X, Wang J, Lin W. mGOF-loc: a novel ensemble learning method for human protein subcellular localization prediction. Neurocomputing. 2016;217:73-82. https://doi.org/10.1016/..neucom.2015.09.137.

34. Wan S, Mak M-W, Kung S-Y. GOASVM: a subcellular location predictor by incorporating term-frequency gene ontology into the general form of Chou's pseudo-amino acid composition. J Theor Biol. 2013;323:40-8.

35. Horton P, Park K-J, Obayashi T, Fujita N, Harada H, Adams-Collier CJ, et al. WoLF PSORT: protein localization predictor. Nucleic Acids Res. 2007;35 suppl_2:W585-7. doi:https://doi.org/10.1093/nar/gkm259.

36. Altschul SF, Gish W, Miller W, Myers EW, Lipman DJ. Basic local alignment search tool. J Mol Biol. 1990;215:403-10.

37. Nielsen H. Predicting Secretory Proteins with SignalP BT - Protein Function Prediction: Methods and Protocols. In: Kihara D, editor. New York, NY: Springer New York; 2017. p. 59-73. doi:https://doi.org/10.1007/978-1-4939-7015-5_6.

38. Krogh A, Larsson B, von Heijne G, Sonnhammer EL. Predicting transmembrane protein topology with a hidden markov model: application to complete genomes. J Mol Biol. 2001;305:567-80. https://doi.org/10.1006/JMBI.2000 4315.

39. Binns D, Dimmer E, Huntley R, Barrell D, O'Donovan C, Apweiler R. QuickGO: a web-based tool for Gene Ontology searching. Bioinformatics. 2009;25:3045-6. https://doi.org/10.1093/bioinformatics/btp536.

40. Keerthikumar S, Chisanga D, Ariyaratne D, Al Saffar H, Anand S, Zhao K, et al. ExoCarta: a web-based compendium of exosomal cargo. J Mol Biol. 2016;428:688-92. https://doi.org/10.1016/J.JMB.2015.09.019.

41. Consortium TU. UniProt: a worldwide hub of protein knowledge. Nucleic Acids Res. 2018;47:D506-15. https://doi. org/10.1093/nar/gky1049. 
42. Li W, Godzik A. Cd-hit: a fast program for clustering and comparing large sets of protein or nucleotide sequences. Bioinformatics. 2006;22:1658-9. https://doi.org/10.1093/bioinformatics/btl158.

43. Olson SA. EMBOSS opens up sequence analysis. European Molecular Biology Open Software Suite. Brief Bioinform. 2002;3:87-91.

44. Frank E, Hall M, Trigg L, Holmes G, Witten IH. Data mining in bioinformatics using Weka. Bioinformatics. 2004;20:2479-81. https://doi.org/10.1093/bioinformatics/bth261.

45. Kandaswamy KK, Pugalenthi G, Kalies K-U, Hartmann E, MartinetzT. EcmPred: prediction of extracellular matrix proteins based on random forest with maximum relevance minimum redundancy feature selection. J Theor Biol. 2013;317:377-83. https://doi.org/10.1016/J.JTBl.2012.10.015.

46. Breiman L. Random forests. Mach Learn. 2001;45:5-32. https://doi.org/10.1023/A:1010933404324.

47. Bhasin M, Reinherz EL, Reche PA. Recognition and classification of histones using support vector machine. J Comput Biol. 2006;13:102-12. https://doi.org/10.1089/cmb.2006.13.102.

48. Bhaskaran R, Ponnuswamy PK. Positional flexibilities of amino acid residues in globular proteins. Int J Pept Protein Res. 1988;32:241-55. https://doi.org/10.1111/j.1399-3011.1988.tb01258.x.

49. Bigelow CC. On the average hydrophobicity of proteins and the relation between it and protein structure. J Theor Biol. 1967;16:187-211.

50. Dayhoff MO, Schwartz RM. Chapter 22: A model of evolutionary change in proteins. In: in Atlas of Protein Sequence and Structure. 1978.

51. Klein P, Kanehisa M, DeLisi C. Prediction of protein function from sequence properties. Discriminant analysis of a data base. Biochim Biophys Acta. 1984;787:221-6.

52. Kubota M, Ooi Y, Obatake M, Kubota Y, Om T. Optimization of amino acid parameters for correspondence of sequence to tertiary structures of proteins. Bull Inst Chem Res Kyoto Univ. 1985::82-94.

53. Zimmerman JM, Eliezer N, Simha R. The characterization of amino acid sequences in proteins by statistical methods. J Theor Biol. 1968;21:170-201.

54. Koehl P, Levitt M. A brighter future for protein structure prediction. Nat Struct Biol. 1999;6:108-11.

55. Mitaku S, Hirokawa T, Tsuji T. Amphiphilicity index of polar amino acids as an aid in the characterization of amino acid preference at membrane-water interfaces. Bioinformatics. 2002;18:608-16.

56. Juretić D, Lučić B, Zucić D, Trinajstić N. Protein transmembrane structure: recognition and prediction by using hydrophobicity scales through preference functions. In: Párkányi CBT-T and CC, editor. Theoretical Organic Chemistry. Elsevier; 1998. p. 405-45. doi:https://doi.org/10.1016/S1380-7323(98)80015-0.

\section{Publisher's Note}

Springer Nature remains neutral with regard to jurisdictional claims in published maps and institutional affiliations.

- fast, convenient online submission

- thorough peer review by experienced researchers in your field

- rapid publication on acceptance

- support for research data, including large and complex data types

- gold Open Access which fosters wider collaboration and increased citations

- maximum visibility for your research: over $100 \mathrm{M}$ website views per year

At BMC, research is always in progress.

Learn more biomedcentral.com/submissions 